\title{
Autobiographical memories of anxiety-related experiences
}

\author{
Amy Wenzel ${ }^{\mathrm{a}, *}$, Keri Pinna ${ }^{\mathrm{b}}$, David C. Rubin ${ }^{\mathrm{c}}$ \\ ${ }^{a}$ Department of Psychology, University of North Dakota, Grand Forks, ND 58202-8380, USA \\ b Department of Psychology, Kent State University, Kent, OH 42242, USA \\ c Department of Psychological and Brain Sciences, Duke University, Durham, NC 27708, USA
}

Received 23 September 2002; received in revised form 1 May 2003; accepted 1 May 2003

\begin{abstract}
Ninety-nine undergraduate students retrieved three memories associated with each of the five emotional experiences: panic, trauma, worry, social anxiety, and feeling content. Subsequently, they answered 24 questions assessing properties of each memory, including the vividness and perceived accuracy of the memories and sensory, emotional, and anxiety-related experiences during retrieval. Memories were coded for affective tone and specificity. Results indicated that panic-related and trauma-related memories were rated similarly as content memories, but that they generally were associated with more imagery and emotional experiencing than worry-related or social anxiety-related memories. Participants experienced panic and worry symptoms to the greatest degree when they retrieved panic-related and trauma-related memories. All anxiety-related memories were characterized by more negative tone than content memories. Panic-related and trauma-related memories were more specific than worry-related, social anxiety-related, and content memories. These findings can explain partially why individuals with some, but not all, anxiety disorders experience enhanced memory for threatening material.
\end{abstract}

(C) 2003 Elsevier Ltd. All rights reserved.

Keywords: Autobiographical memory; Anxiety disorder; Panic; Trauma; Worry; Social anxiety

\section{Introduction}

According to cognitive theories of anxiety disorders, anxious individuals process threat-related information in a biased manner (e.g., Beck \& Clark, 1997; Rapee \& Heimberg, 1997; Williams et al., 1997). Empirical work has provided robust evidence confirming that anxious individuals allocate their attention toward threat-related stimuli at the expense of neutral stimuli (e.g., Mogg \&

\footnotetext{
* Corresponding author. Tel.: +1-701-777-4496; fax: +1-701-777-3454.

E-mail address: amy_wenzel@und.nodak.edu (A. Wenzel).
} 
Bradley, 1998) and interpret ambiguous information as if it were dangerous (e.g., MacLeod \& Cohen, 1993). On the other hand, evidence for enhanced memory for threat-related material in anxiety disorders is equivocal. Studies that examine memory for threat-related material in individuals with panic disorder (PD) and individuals with posttraumatic stress disorder (PTSD) generally find evidence for memory biases toward threat-related stimuli (e.g., McNally, Foa, \& Donnell, 1989; Paunovic, Lundh, \& Ost, 2002). In contrast, studies that examine memory for threat-related and neutral material in individuals with generalized anxiety disorder (GAD) and individuals with social phobia (SP) generally do not find evidence for such a bias (e.g., Mogg, Mathews, \& Weinman, 1987; Rapee et al., 1994). However, there are several exceptions to this pattern of findings (e.g., Amir, Foa, \& Coles, 2000; Lundh \& Ost, 1996), and confounding variables, such as the presence of comorbid depressive symptoms (e.g., Becker et al., 1999), further complicate interpretation of results.

One explanation for discrepant findings in this area is that the explicit and implicit memory paradigms used in most studies in this literature are not sensitive enough to activate relevant fear structures to induce cognitive biases in some anxiety disorders (cf. Burke \& Mathews, 1992; Wenzel \& Holt, 2002). That is, memory tasks using single words may not adequately capture the amount of threat that would create a sense of danger or discomfort and would in turn bias the sequence of information processing. This explanation runs contrary to robust findings in the literature examining attentional biases in anxiety disorders. Although single words are adequate to activate fear structures in tasks that require relatively shallow processing such as those used to assess attentional biases (e.g., MacLeod, Mathews, \& Tata, 1986), tasks assessing memory biases may require more elaborate processing (cf. Mogg et al., 1987). It is possible that stimuli for these memory tasks need to resemble more closely the type of threat that is encountered in the everyday lives of individuals with anxiety disorders.

To address this methodological concern, many researchers have adopted autobiographical memory tasks to examine memories of events that anxious individuals have actually experienced. Autobiographical memory is the recollection of one's personal experiences that incorporates verbal description, mental imagery, and often emotional experiencing (Rubin, 1996). However, it is also difficult to draw definitive conclusions about the nature of anxiety-related memories in this literature. For example, individuals with GAD demonstrate enhanced memory for threat when autobiographical memory is assessed with some, but not all dependent variables (Burke \& Mathews, 1992; Richards \& Whittaker, 1990). Results from several investigations suggest that SP is not associated with enhanced retrieval of social threat memories (Rapee et al., 1994; Wenzel, Jackson, \& Holt, 2002). Autobiographical memories of threat-related experiences have not been examined to date in individuals with PD. Although two investigations have found that PTSD is associated with difficulty in retrieving specific positive autobiographical memories (McNally et al., 1994, 1995), threat-related stimuli were not included in these designs. Thus, autobiographical memory is a promising method that has the potential to clarify the nature of memory biases in particular anxiety disorders, but additional research is needed to systematically investigate threatrelated autobiographical memories in a similar manner across anxiety pathologies.

In addition, researchers have assumed that differences in specific anxiety-related pathologies and/or differences in methodologies account for the mixed findings in the anxiety and memory literature. However, one explanation that has not yet been considered is that the nature of some types of anxiety-related memories is inherently different than the nature of other types of anxiety- 
related memories. The pattern of findings that PD and PTSD, but not GAD and SP, are characterized by a memory bias toward threat could have emerged because panic- and trauma-related memories have properties that make them more salient, detailed, and/or vivid than memories related to worry and social anxiety. This explanation suggests that an interaction between specific pathologies (i.e., PD, PTSD) and the nature of the memories (i.e., panic-related, trauma-related) would account for memory biases toward threat in these two populations, rather than only pathology status per se.

To address this issue, the present study was designed to investigate properties of four types of anxiety-related autobiographical memories-those related to the experiences of panic, trauma, worry, and social anxiety. This study represents an advance in the literature, as memories of these four types of anxiety-related experiences have not been considered in one study. A sample of individuals representing a normal population was recruited so that properties of these memories could be examined independent of biases that might characterize individuals with anxiety pathology. Participants were instructed to retrieve specific autobiographical memories associated with these four types of anxiety experiences as well with the experience of feeling content, which served as a comparison condition. Next, participants rated a series of items to characterize the vividness and accuracy of these memories, the associated sensory and emotional experiences, and the anxiety responses experienced when retrieving these memories (cf. Berntsen, Willerts, \& Rubin, in press; Rubin, Feldman, \& Beckham, in press; Rubin, Schrauf, \& Greenberg, in press; Sheen, Kemp, \& Rubin, 2001).

It was expected that panic- and trauma-related memories would be distinguished from worryand social anxiety-related memories on all items assessing vividness, perceived accuracy, and associated sensory and emotional experiences but that memories within these broad groupings would not be distinguished from each other. Further, it was predicted that each of the four groups could be differentiated on the particular anxiety symptoms experienced during retrieval (i.e., panic-related memories would be accompanied to the greatest degree by panic-related sensations).

\section{Method}

\subsection{Participants}

Participants were 99 undergraduate psychology students who received course or extra credit for completing the study. They had a mean age of $19.8(\mathrm{SD}=2.2), 66.7 \%$ were female, and 93.9\% were Caucasian.

\subsection{Autobiographical memory questionnaire}

The 24-item autobiographical memory questionnaire (AMQ) was modeled after that described by Rubin, Schrauf et al. (in press). All items were rated on a seven-point Likert-type scale, with most anchors ranging from 1 (not at all) to 7 (more than any other memory). The first 12 items were questions that Rubin et al. used to assess properties of general autobiographical memories in their sample, such as the vividness and accuracy of the memories and sensory and emotional experiences during retrieval (e.g., while remembering the event, I feel as though I am reliving 
the event). The remaining 12 items were created for the purpose of the present study. They measured the degree to which participants experienced four domains of anxiety symptoms - panic, traumatic stress, worry, and social anxiety-during retrieval of the memories (e.g., while remembering the event, I feel embarrassed).

\subsection{Procedure}

Participants were tested in large group testing sessions. First, they were provided with a set of instructions describing the procedure, defining the construct of autobiographical memory, and explaining how to retrieve specific memories. Next, participants recorded three specific memories for each of five categories: (a) situations in which they experienced panic, (b) situations in which they experienced trauma, (c) situations in which they experienced worry, (d) situations in which they experienced social anxiety, and (e) situations in which they were content. For each of these categories, participants received a description of what was meant by these constructs. Subsequently, they recorded a two- or three-word phrase to describe each of the first three memories that came to mind in association with the category name. For each of the three memories retrieved, they wrote a more lengthy description of the memory and completed the series of 24 questions assessing properties of the memory. Participants retrieved memories and answered associated questions in one category before moving onto the next category. Participants retrieved memories associated with each category in one of five orders of presentation determined by a Latin Square counterbalancing scheme.

\subsection{Coding}

Memories were coded for affective tone and their specific vs. general nature, two variables commonly considered in clinically relevant investigations of autobiographical memory in pathology groups (e.g., Wenzel et al., 2002). For affective tone, two trained coders classified each memory into one of five categories: (a) positive affect, (b) positive tone, (c) neutral, (d) negative tone, or (e) negative affect (cf. Wenzel et al., 2003). Memories assigned affect codes included specific references to affect that they either experienced at the time of the situation or during retrieval. For example, memories coded as reflecting positive affect made specific references to experiences such as pride, enthusiasm, and alertness, whereas memories coded as reflecting negative affect made specific references to experiences such as fear, anger, and irritation (Watson, Clark, \& Tellegen, 1988). In contrast, memories assigned tone codes clearly described an experience that most people would regard as positive or negative but that did not make reference to a specific affective experience. Responses that had no discernible valence were given ratings of neutral. Coders were trained on eight cases and reached acceptable reliability (kappa $=0.75$ ). Coders attended a reliability meeting once a week to prevent bias and drift.

The second coding scheme required two trained coders to rate each memory as being either specific or general. A memory was coded as specific if it referred to a discrete event that occurred during a period of time of no more than 1 day (cf. Williams \& Broadbent, 1986). A memory was coded as general if it contained one or more of the following attributes: (a) there was not enough information provided to discern whether the person was referring to a discrete event that occurred in a 1 day period (e.g. "studying for an exam"); (b) an event that took place over the 
course of more than 1 day (e.g. "summer vacation"); or (c) a recurrent event (e.g. "every time I have to give a speech"). Coders were trained on 18 cases and obtained acceptable reliability $($ kappa $=0.72)$. Coders attended a reliability meeting once a week to prevent bias and drift.

\subsection{Data analysis}

\subsubsection{Analytic strategy}

Because theoretical and empirical work suggests that PD and PTSD are associated with different patterns of memory biases than are GAD and SP, responses were collapsed in these two general categories and analyzed using an omnibus repeated measures ANOVA with three levels (panic/trauma, worry/social anxiety, content). Subsequently, planned comparisons between the panic-related and trauma-related memories and between the worry-related and social anxietyrelated memories were conducted for all variables that significantly differentiated between the general categories in the omnibus analyses. Only those planned comparisons that were significant are reported.

\subsubsection{Dependent variables}

Each of the 12 items from Rubin, Feldman et al. (in press), the affective tone coding scheme, and the specific vs. general coding scheme were analyzed separately. In contrast, the 12 items assessing anxiety symptoms during retrieval that were created for the present study were collapsed into four broad categories corresponding to the particular anxiety domain to which they were related, as defined by symptoms associated with these specific anxiety disorders in the DSM-IV (American Psychiatric Association, 1994). That is, four dependent variables were created that reflected symptoms of panic, traumatic stress, generalized anxiety, and social anxiety.

\section{Results}

Table 1 summarizes the means for the dependent variables of interest in this study. In addition, $F$ values for the omnibus three-level repeated measures ANOVA and their corresponding values of statistical significance are presented.

\subsection{Feeling of recollection}

Two items measured the degree to which participants experienced a feeling of recollection when they retrieved their autobiographical memories: "While remembering the event, I feel as though I am reliving the original event" and "While remembering the event, I feel as though I am there again" (Rubin, Feldman et al., in press). Both of these items significantly discriminated between groups of memories, with worry/social anxiety-related memories rated lower on these dimensions than panic/trauma-related memories and content memories. Planned comparisons for both variables revealed that trauma-related memories were rated higher than panic-related memories $(p=0.031$ and 0.001 , respectively) but that there were no differences between the worryrelated and social anxiety-related memories. 


\subsection{Imagery value of memories}

Four items measured aspects of the imagery associated with participants' autobiographical memories, hearing the event in one's mind, seeing the event in one's mind, remembering the setting in which it occurred, and being able to recreate the details of the event, and all yielded significant differences between groupings of memories. The general pattern of differences for these four questions was that panic/trauma-related memories did not differ from content memories, but that both of these categories of memories were rated higher than the worry/social anxietyrelated memories. Planned comparisons for the recreating details item indicated higher ratings for trauma-related memories than for panic-related memories $(p<0.001)$ and higher ratings for worry-related memories than for social anxiety-related memories $(p<0.001)$. Planned comparisons for remembering the setting item indicated higher ratings for social anxiety-related than worry-related memories $(p<0.001)$. There were no differences between specific anxiety-related memories on the seeing and hearing items.

\subsection{Emotions experienced during retrieval}

Four items measured aspects of the emotional experiencing associated with participants' autobiographical memories: feeling the particular emotions felt during the event, experiencing the emotions as strongly as during the event, experiencing extremely positive emotions during retrieval, and experiencing extremely negative emotions during retrieval. Each of these items significantly discriminated between groups of memories. However, the pattern of differences between categories of memories depended on the specific item.

For ratings of the degree to which participants felt the particular emotions during the event, panic/trauma related memories did not differ from content memories, but both types of memories were rated higher on this dimension than worry/social anxiety-related memories. In contrast, for ratings of emotion strength, panic/trauma-related memories were rated as having stronger emotion than content memories, which in turn were rated as having stronger emotion than worry/social anxiety-related memories. Planned comparisons for both of these variables indicated that ratings were higher for the trauma-related memories than for the panic-related memories $(p<0.001)$ but that there were no differences between the worry-related and social anxiety-related memories.

Content memories were associated with more positive emotion than panic/trauma related memories, which in turn were associated with more positive emotion than worry/social anxiety-related memories. Planned comparisons suggested that social anxiety-related memories were associated with higher ratings of positive emotion than were worry-related memories $(p<0.001)$ but that panic-related and trauma-related memories did not differ on this variable. Panic/trauma-related memories were associated with more negative emotion than worry/social anxiety-related memories, which in turn were associated with more negative emotion than content memories. Planned comparisons indicated that trauma-related memories were associated with higher levels of negative emotion than were panic-related memories $(p<0.001)$, and worry-related memories were associated with higher levels of negative emotion than were social anxiety-related memories $(p<$ $0.001)$. 


\subsection{Perceived accuracy}

Two questions measured the degree to which participants believed their memories were accurate: "To what extent do you believe that this event occurred as you remembered it?" and "How hard would it be to convince you that this memory did not occur as you remembered it?" (Rubin, Schrauf et al., in press). Only the first item significantly discriminated between groups of memories. Although the panic/trauma-related memories did not differ from content-related memories on this dimension, both types of memories were associated with a stronger belief that the memory occurred exactly as remembered than worry/social anxiety-related memories. Planned comparisons revealed that trauma-related memories were associated with a higher level of belief than panicrelated memories $(p=0.038)$ but that worry-related and social anxiety-related memories did not differ on this dimension.

\subsection{Anxiety-related symptoms during retrieval}

The omnibus ANOVAs were highly significant for each domain of anxiety symptoms experienced during retrieval. For panic and worry symptoms, panic/trauma-related memories were rated higher than worry/social anxiety-related memories, which in turn were rated higher than content memories. Planned comparisons suggested that worry-related memories were associated with higher levels of worry symptoms than social anxiety-related memories $(p<0.001)$ and that trauma-related memories were associated with higher levels of worry symptoms than panic-related memories $(p=0.013)$. There were no differences between specific anxiety-related memories in the levels of panic symptoms experienced during retrieval. In contrast, for traumatic stress and social anxiety symptoms, both panic/trauma memories and worry/social anxiety-related memories were rated higher than content memories but were not different from each other. Planned comparisons for social anxiety symptoms revealed higher ratings for panic-related memories than for trauma-related memories $(p=0.014)$ and higher ratings for social anxiety-related memories than for worry-related memories $(p=0.001)$. There were no differences between specific anxietyrelated memories in the levels of traumatic stress symptoms experienced during retrieval.

\subsection{Affective tone}

Each of the four major affect codes (i.e., positive affect, positive tone, negative affect, negative tone) significantly discriminated between groups of memories. Participants retrieved more content memories that were coded as positive affect and as positive tone than panic/trauma-related and worry/social anxiety-related memories. There was no difference between the broad classes of anxiety-related memories for positive affect codes, but participants retrieved more worry/social anxiety-related memories than panic/trauma-related memories that were coded as positive tone. Planned comparisons failed to uncover any differences in positive tone ratings between specific types of anxiety-related memories.

In addition, participants retrieved more panic/trauma-related and worry/social anxiety-related memories than content memories associated with both negative affect and negative tone. Although there was a greater number of panic/trauma-related memories characterized by negative tone than worry/social anxiety-related memories, there were more worry/social anxiety-related memories 
than panic/trauma-related memories that were characterized by negative affect. Planned comparisons indicated that participants retrieved a greater number of trauma-related memories than panicrelated memories that were characterized by negative tone $(p<0.001)$, and they retrieved a greater number of worry-related memories than social anxiety-related memories that were characterized by negative tone $(p=0.001)$. Planned comparisons failed to uncover any differences in negative affect ratings between specific types of anxiety-related memories.

\subsection{Specificity}

There was a significant difference between groups of memories in the percentage of memories coded as specific. Follow-up analyses revealed that participants retrieved a greater number of specific panic/trauma-related memories than worry/social anxiety-related memories and content memories, but there was no difference in the specificity of the latter two types of memories. Planned comparisons revealed that participants retrieved more specific panic-related memories than trauma-related memories $(p=0.017)$ and more specific social anxiety-related memories than worry-related memories $(p<0.001)$.

\section{Discussion}

The present study was conducted to examine a possible factor to explain a general pattern that has emerged in the anxiety disorders literature, such that individuals with PD or PTSD, but not necessarily individuals with GAD or SP, are characterized by enhanced memory for threat-related information. To achieve this aim, a sample representing the normal population of undergraduate psychology students retrieved memories associated with each of these anxiety domains and rated their memories on a number of dimensions. On 17 of 21 dependent variables, panic-related and trauma-related memories were rated differently than worry-related and social anxiety-related memories. For all variables except percentage of negative affect codes, differences were in the direction that panic- and trauma-related memories were more vivid, detailed, and specific, were believed to be more accurate, and were accompanied by stronger sensory and emotional experiences than worry-related and social anxiety-related memories. Although the worry-related memories differed from the social anxiety-related memories in only a few cases, the trauma-related memories differed from the panic-related memories in 10 cases. In general, trauma-related memories were more vivid and more likely to be accompanied by intense negative emotions than panic-related memories.

Although results generally supported our first hypothesis, our expectation that participants would experience diagnosis-specific anxiety symptoms during retrieval of memories from the different anxiety domains was not supported. Instead, the retrieval of all types of anxiety-related memories was associated with symptoms of traumatic stress and social anxiety to a greater degree than the retrieval of content memories, and panic/trauma-related memories were associated with the greatest degree of panic and worry symptoms. Although the questions were carefully constructed based on DSM-IV (American Psychiatric Association, 1994) criteria associated with these disorders, in reality, most anxiety symptoms reflect components of general distress that are relevant to many or all anxiety disorders (Zinbarg \& Barlow, 1996). Thus, although the questions were grouped into those that were panic-related, traumatic stress-related, generalized anxiety- 
related, and social anxiety-related, it is likely that they pertained to all four pathologies at least to some degree. The distinction between these four dependent variables, then, might be fairly arbitrary.

In all, this study provides robust evidence suggesting that many properties of panic-related and trauma-related memories are different than the nature of worry-related memories and social anxiety-related memories. To date, most researchers have focused on differences in the specific pathologies to explain the different patterns of memory for threatening information that characterize this literature. It is argued here that properties of the memories themselves, in addition to the specific pathology of the anxious individual, may be an important factor in explaining the mixed results in the literature. At least one theory of anxiety suggests that anxious individuals avoid elaborate processing of threatening material, which subsequently affects their memory for that material (Mogg et al., 1987). It is possible that the vivid nature of panic-related and trauma-related memories interacts with aspects of anxiety pathology to supercede this avoidance mechanism and drive exaggerated memories for these threatening experiences, a process that is not at work for worry-related and social anxiety-related memories.

However, an equally plausible explanation for these results is not that the panic-related and trauma-related memories are particularly salient, but that worry-related and social anxiety-related memories lack the usual sense of recollection, accompanying imagery, intense emotional experiencing, and belief that the memory is accurate. In most cases, results from items assessing these general properties of autobiographical memories suggest that panic-related and trauma-related memories were rated in a similar manner as content memories, and both were rated higher than the worry-related and social anxiety-related memories. These findings replicate data reported by Berntsen (2001), who found that traumatic and extremely happy involuntary autobiographical memories were rated similarly on a number of dimensions. In addition, this finding fits logically with research suggesting that worry is a primarily semantic activity that diverts an individual from the full imaginal and affective experience of anxiety (Borkovec, 1994). These results are also consistent with theoretical models that distinguish between different types of anxiety-related expressions. For example, Barlow (2002) recently outlined etiological models of each of these four anxiety pathologies, and he indicated that the fear response is more characteristic of PD and PTSD than GAD and generalized SP, whereas the construct of anxious apprehension is particularly useful in capturing the experience of individuals with the latter two pathologies. It is possible that fear and anxious apprehension exert effects on memory either to different degrees or through different mechanisms.

Additionally, it should be mentioned that it could be that the events themselves associated with panic and trauma memories are more unexpected, fear-provoking, and accompanied by detail than the events associated with worry and social anxiety. That is, differences in the nature of the encoded events, but not necessarily in the resulting autobiographical memories, could account for the pattern of findings reported in the present study. In the extreme, because worry is a primarily cognitive event characterized by a catastrophic future orientation, it is possible that a greater percentage of worry-related memories are imagined (vs. real), which could result in fewer accompanying details and a diminished sense of recollection. It will be important for future research to identify the properties of the events behind the memories in order to investigate this possibility. Regardless of whether the properties of the events, the memories, or both differentiate panic and trauma-related recollections from worry- and social anxiety-related recollections, the 
fact remains that there are factors in addition to pathology status that account for memory biases observed in some, but not in all anxiety disorders.

One caveat of this design should be noted. The sample comprised undergraduate students who were not selected to be anxious on any of these dimensions. This design choice was made to isolate the properties of the anxiety-related memories independent of individual pathology. However, it is possible that worry-related memories and social anxiety-related memories might have been rated in a similar manner as panic-related and trauma-related memories in individuals who are experiencing GAD and SP at a clinically significant level of severity. Although this possibility is contrary to the proposed mechanism described above to explain the dissociation among anxiety pathologies in their associated memory biases, it is an empirical question that is easily investigated by replicating this design in samples of individuals who are diagnosed with the these anxiety disorders. Moreover, because nearly all individuals who participated in this study were in the 1822 year-old age range, it will be important to replicate these results with a sample of individuals representing the full age range that characterizes the adult population of individuals with anxiety disorders.

In all, results from this study have important implications for the literature on memory biases in anxiety disorders. They suggest that, in addition to carefully assessing the properties of stimuli included into memory tasks, researchers also must ensure that properties of the memories themselves are similar before drawing any conclusions about memory biases in clinical populations. The design introduced in this study has the potential to clarify theory elucidating the mechanism by which memory biases arise in anxiety pathology. However, it is important to link theory with applied issues, and it will be incumbent upon future researchers to map the manner in which retrieval (or avoidance) of these anxiety-related memories exacerbates anxiety symptoms, affects the course of treatment, and predicts relapse.

\section{Acknowledgements}

The authors would like to thank Melanie Goyette and Erin Haugen for their assistance with this research. The autobiographical memory questionnaire is available from the first author upon request.

\section{References}

American Psychiatric Association (1994). Diagnostic and statistical manual of mental disorders (fourth ed.). Washington, DC: American Psychiatric Association.

Amir, N., Foa, E. B., \& Coles, M. E. (2000). Implicit memory bias for threat-relevant information in individuals with generalized social phobia. Journal of Abnormal Psychology, 109, 713-720.

Barlow, D. H. (2002). Anxiety and its disorders: The nature and treatment of anxiety and panic (second edition). New York: Guilford Press.

Beck, A. T., \& Clark, D. M. (1997). An information processing model of anxiety: automatic and strategic processes. Behaviour Research and Therapy, 35, 49-58.

Becker, E. S., Roth, W. T., Andrich, M., \& Margraf, J. (1999). Explicit memory in anxiety disorders. Journal of Abnormal Psychology, 108, 153-163. 
Berntsen, D. (2001). Involuntary memories of emotional events: do memories of traumas and extremely happy memories differ? Applied Cognitive Psychology, 15, S135-S158.

Berntsen, D., Willerts, M., \& Rubin, D. C. (in press). Splintered memories or vivid landmarks? Reliving and coherence of traumatic memories in PTSD. Applied Cognitive Psychology.

Borkovec, T. D. (1994). The nature, function, and origins of worry. In G. C. L. Davey, \& F. Tallis (Eds.), Worry: Perspectives on theory, assessment, and treatment. New York: Wiley.

Burke, M., \& Mathews, A. (1992). Autobiographical memory and clinical anxiety. Cognition and Emotion, 6, 23-35.

Lundh, L. G., \& Ost, L. G. (1996). Recognition bias for critical faces in social phobics. Behaviour Research and Therapy, 34, 787-794.

MacLeod, C., \& Cohen, I. L. (1993). Anxiety and the interpretation of ambiguity: a text comprehension study. Journal of Abnormal Psychology, 102, 238-247.

MacLeod, C., Mathews, A., \& Tata, P. (1986). Attentional bias in the emotional disorders. Journal of Abnormal Psychology, 95, 15-20.

McNally, R. J., Foa, E. B., \& Donnell, C. D. (1989). Memory bias for anxiety information in patients with panic disorder. Cognition and Emotion, 3, 27-44.

McNally, R. J., Lasko, N. B., Macklin, M. L., \& Pitman, R. K. (1995). Autobiographical memory disturbance in combat-related posttraumatic stress disorder. Behaviour Research and Therapy, 33, 619-630.

McNally, R. J., Litz, B. T., Prassas, A., Shin, L. M., \& Weathers, F. W. (1994). Emotional priming of autobiographical memory in post-traumatic stress disorder. Cognition and Emotion, 8, 351-367.

Mogg, K., \& Bradley, B. P. (1998). A cognitive-motivational analysis of anxiety. Behaviour Research and Therapy, $36,809-848$.

Mogg, K., Mathews, A., \& Weinman, J. (1987). Memory bias in clinical anxiety. Journal of Abnormal Psychology, 96, 94-98.

Paunovic, N., Lundh, L. G., \& Ost, L. G. (2002). Attentional and memory bias for emotional information in crime victims with posttraumatic stress disorder (PTSD). Journal of Anxiety Disorders, 16, 675-692.

Rapee, R. M., \& Heimberg, R. G. (1997). A cognitive-behavioral model of anxiety in social phobia. Behaviour Research and Therapy, 35, 741-756.

Rapee, R., McCallum, S. L., Melville, L. F., Ravenscroft, H., \& Rodney, J. M. (1994). Memory bias in social phobia. Behaviour Research and Therapy, 29, 317-323.

Richards, A., \& Whittaker, T. M. (1990). Effects of anxiety and mood manipulation in autobiographical memory. British Journal of Clinical Psychology, 29, 145-153.

Rubin, D.C. (Ed.). (1996). Remembering our past: Studies in autobiographical memory. Cambridge, England: Cambridge University Press.

Rubin, D. C., Feldman, M. E., \& Beckham, J. C. (in press). Reliving, emotions, and fragmentation in the autobiographical memories of veterans diagnosed with PTSD. Applied Cognitive Psychology.

Rubin, D. C., Schrauf, R. W., \& Greenberg, D. L. (in press). Remembering, reliving, and believing autobiographical memories: the method of inter- and intra-individual correlations. Memory and Cognition.

Sheen, M., Kemp, S., \& Rubin, D. C. (2001). Twins dispute memory ownership: a new false memory phenomenon. Memory and Cognition, 29, 779-788.

Watson, D., Clark, L. A., \& Tellegen, A. (1988). Development and validation of a brief measure of positive and negative affect: the PANAS scales. Journal of Personality and Social Psychology, 54, 1063-1070.

Wenzel, A., \& Holt, C. S. (2002). Memory bias against threat in social phobia. British Journal of Clinical Psychology, $41,73-79$.

Wenzel, A., Jackson, L. C., Brendle, J. R., \& Pinna, K. (2003). Autobiographical memories associated with feared stimuli in fearful and nonfearful individuals. Anxiety, Stress and Coping: An International Journal, 16, 1-15.

Wenzel, A., Jackson, L. C., \& Holt, C. S. (2002). Social phobia and the recall of autobiographical memories. Depression and Anxiety, 15, 186-189.

Williams, J. M. G., \& Broadbent, K. (1986). Autobiographical memory in suicide attempters. Journal of Abnormal Psychology, 93, 144-149.

Williams, J. M. G., Watts, F. N., MacLeod, C., \& Mathews, A. (1997). Cognitive psychology and emotional disorders, 2nd ed. New York: Wiley. 
Zinbarg, R. E., \& Barlow, D. H. (1996). Structure of anxiety and the anxiety disorder: a hierarchical model. Journal of Abnormal Psychology, 105, 181-193. 